\title{
Does preoperative computed tomography scanning aid assessment of oesophageal carcinoma?
}

\author{
S.J. Kirk' ${ }^{1}$, R.J. Moorehead ${ }^{1}$, E. McIlrath ${ }^{2}$, J.P. Gibbons ${ }^{3}$ and R.A.J. Spence' \\ ${ }^{1}$ Department of Surgery, Belfast City Hospital, ${ }^{2}$ Department of Radiology, Royal Victoria Hospital and \\ ${ }^{3}$ Department of Thoracic Surgery, Royal Victoria Hospital, Belfast, $N$. Ireland.
}

\begin{abstract}
Summary: In this study 50 patients (36 male, 14 female) with oesophageal carcinoma have been examined preoperatively by computed tomography. Three parameters were assessed, nodal involvement, invasion of adjacent organs, and metastatic disease. For all patients the computed tomography findings were correlated with the subsequent surgical and pathological findings. Sensitivity for both invasion and node involvement is low (36\% to $67 \%)$. Specificity however, is high ( $85 \%$ to $95 \%)$. Computed tomography is therefore relatively reliable when it predicts a 'negative result' for either invasion or node involvement. However, its predictive value for a positive result is not as reliable.
\end{abstract}

\section{Introduction}

Carcinoma of the oesophagus has a poor prognosis. It is often well advanced with local invasion, nodal involvement and occasionally distant metastases, by the time the patient first presents. ${ }^{1,2}$

In patients considered suitable for surgery perioperative mortality for oesophageal resection varies from $30 \%$ to $6 \%^{3,4}$ and the overall 5 year survival is seldom better than $15 \% .^{4}$ However, survival figures vary significantly with stage. For patients with node involvement at operation the 3 year survival figures are $25 \%$ for squamous cell and $18 \%$ for adenocarcinoma. ${ }^{5}$ It would therefore seem reasonable to avoid unnecessary exploratory surgery and to operate only on those patients in whom a cure, or where good palliation, ${ }^{6}$ can be achieved.

The role of computed tomography (CT) scanning in assessment of pulmonary and mediastinal tumours has been previously evaluated, and the data suggest that it yields more useful information than other conventional radiological techniques (plain X-ray, barium meal, ultrasonography and radio-isotope scanning. ${ }^{7,8}$ We therefore conducted a prospective study to determine the value of computed tomography in the preoperative assessment of patients with oesophageal carcinoma.

\section{Patients and methods}

Patients were entered into the study if they were clinically considered suitable for surgery. All had

Correspondence: R.A.J. Spence, M.D., F.R.C.S., Level 2, Belfast City Hospital, Lisburn Road, Belfast, $\mathrm{N}$. Ireland.

Accepted: 21 September 1989 histologically proven carcinoma of the oesophagus. All patients underwent surgery, giving surgical and pathological correlation with our preoperative findings.

Fifty patients were studied, 36 male, 14 female. Age range was $42-81$ years, mean age 67 years. Thirty-two patients had adenocarcinoma, 15 had squamous cell tumours, 2 had anaplastic carcinoma and one patient was diagnosed as primary melanoma of the oesophagus. Tumours were located in the lower third of the oesophagus in 35 patients $(70 \%)$, in the middle third in $13(26 \%)$, and in the upper third in $2(4 \%)$.

All patients underwent oesophagoscopy. The distance of the tumour from the incisors was measured and if the tumour did not prevent passage of the endoscope, the length and distal extent of tumour was recorded. Barium meal was performed in 36 patients and 11 had bronchoscopy. All patients had abdominal ultrasonography (Diasonics), looking specifically for ascites and metastatic liver involvement.

A CT protocol based on the work of Picus and others $^{9}$ was established. Two scanning machines were used (a Siemens Somatom DR3 and a DR2), and $8 \mathrm{~mm}$ contiguous scans (using the rev sen facility) taken at the level of the tumour. Above and below the tumour level $8 \mathrm{~mm}$ images were recorded with $1.2 \mathrm{~mm}$ intervals, scans were usually terminated at the lower border of the liver. Oral contrast media (E-Z-Cat Barium) was given routinely at 5 minutes and zero minutes prior to commencement of the scan.

The following characteristics were recorded. To assess invasion we noted circumference of contact between tumour and aorta (greater than 90 degrees 
indicating invasion), displacement and/or compression of adjacent organs and loss of tissue/fat planes between the oesophagus and contiguous organs. Lymph node enlargement ( $5 \mathrm{~mm}$ or greater for mediastinum; $10 \mathrm{~mm}$ or greater in abdomen), was the criterion chosen to assess nodal involvement. Regional node fields were assessed at, above and below the tumour level. The liver was also examined for the presence of metastatic disease. Intravenous contrast medium was not given routinely but was used in 2 cases of doubtful invasion.

CT findings were correlated with the subsequent surgical and pathological findings. Surgically, organ invasion was defined as tumour adherence which could not be dissected free by gentle, cautious dissection. All submitted nodes were examined for metastatic involvement.

All patients had surgery, one patient had no definitive procedure other than laparotomy, 7 patients were intubated (Celestin tube), 13 had laparotomy and right thoracotomy (Ivor-Lewis procedure) and the remaining 29 patients had an oesophagogastrectomy using a left thoracoabdominal approach.

\section{Results}

\section{Invasion}

Above the diaphragm CT suggested that 5 patients had evidence of invasion, which was surgically confirmed in 2 patients (tracheal and pericardial invasion). It was thought that $\mathbf{4 5}$ patients had no evidence of invasion on CT and this was correct in 42 cases. The false negative assessments arose in 2 patients with aortic invasion and in one patient with pericardial involvement. Sensitivity and spe- cificity above the diaphragm were $40 \%$ and $93 \%$ respectively (Table I). Below the diaphragm 4 patients were incorrectly assessed by CT. In 3 cases errors were related to peridiaphragmatic invasion and in one to pancreatic invasion giving a sensitivity of $67 \%$ and a specificity of $95 \%$ (Table I).

\section{Node status}

Above the diaphragm 43 patients had negative nodes on CT. Histology, however, demonstrated node involvement in 7 of these patients. Seven patients were thought to have positive nodes on CT, but this was found to be correct in only 4 patients.

Below the diaphragm CT suggested that 34 patients had negative nodes, which was subsequently shown to be true in 17 of these patients. Sixteen patients were pre-operatively thought to have positive nodes and this was histologically correct in 13 patients (Table II). Node size was not measured by the pathologist, but of the 17 patients whose nodes were histologically positive yet CT negative, 14 had only microscopic evidence of metastatic node involvement.

\section{Extra-nodal disease}

At surgery 6 patients had evidence of extra-nodal, metastatic disease. CT did not diagnose this in 3 patients (2 with liver involvement and 1 with ascites). However, it correctly excluded metastatic disease in the remaining 44 patients. The ultrasound scan also missed metastatic disease in 2 of these 6 patients.

Separate analysis was performed on the 35 patients who had tumours confined to the lower oesophagus and gastro-oesophageal junction. This shows similar results for invasion and nodal

Table I Invasion

\begin{tabular}{llcc}
\hline & & \multicolumn{2}{c}{ CT assessment } \\
\cline { 3 - 4 } & & Positive & Negative \\
\hline Above diaphragm & Positive & 2 & 3 \\
Surgical findings & Negative & 3 & 42 \\
& Sensitivity & $40 \%$ & \\
& Specificity & $93 \%$ & \\
& Predictive value & 0.40 & \\
Below diaphragm & (for positive result) & & \\
Surgical findings & Positive & 4 & 2 \\
& Negative & 2 & 42 \\
& Sensitivity & $67 \%$ & \\
& Specificity & $95 \%$ & \\
& Predictive value & 0.66 & \\
& (for positive result) & & \\
\hline
\end{tabular}


Table II Nodal involvement

\begin{tabular}{llcc}
\hline & & \multicolumn{2}{c}{ CT assessment } \\
\cline { 3 - 4 } & & Positive & Negative \\
\hline Above diaphragm & Positive & 4 & 7 \\
Pathology findings & Negative & 3 & 36 \\
& Sensitivity & $36 \%$ & \\
& Specificity & $92 \%$ & \\
& Predictive value & 0.57 & \\
Below diaphragm & (for positive value) & 13 & 17 \\
Pathology findings & Nesitive & 3 & 17 \\
& Segative & $43 \%$ & \\
& Sensitivity & $85 \%$ & \\
& Specificity & 0.81 & \\
& (for positive result) & & \\
\hline
\end{tabular}

involvement, above and below the diaphragm. Above the diaphragm, for nodes and invasion, sensitivity was $40 \%$ and $30 \%$ and specificity was $96 \%$ and $97 \%$ respectively. Below the diaphragm sensitivity was $52 \%$ and specificity was $80 \%$ for nodes. The values for invasion were sensitivity $80 \%$ and specificity $96 \%$ (Table III).

\section{Discussion}

We believe that for CT to be of value in the preoperative assessment of patients with oesophageal cancer it should be able to separate patients into 2 main surgical categories, resectable, and unresectable, leaving only a small number of patients in an equivocal or indeterminate category regarding operability. This is an important role which if valid would influence patient management, morbidity and mortality, and would also have economic implications. ${ }^{10}$ The surgical management of those patients in the resectable/ unresectable group would be clearly defined. Those patients in the indeterminate group should not be denied the possibility of cure whether by surgery and/or adjuvant radiotherapy/chemotherapy.

Technically resectable tumours, even in the presence of nodal involvement, are probably best managed surgically both in terms of palliation and potential cure. However, for hepatic metastatic disease, or for patients with significant invasion, laser or intubation procedures for the symptomatic patient are best, and generally no procedure is required for the asymptomatic patient. ${ }^{11}$

In our study metastatic disease was missed in half of patients in whom it was present (3 of 6), but accurately excluded in the remaining 44 patients. Although our numbers are small this is disappointing; two of the patients had diffuse micrometa-
Table III Sites of invasion

\begin{tabular}{lcc}
\hline Invasion & $\begin{array}{c}\text { Sites of false } \\
\text { positive results }\end{array}$ & $\begin{array}{c}\text { Sites of false } \\
\text { negative results }\end{array}$ \\
\hline Aorta & $11(2)$ & $11(2)$ \\
Pericardium & 1 & 1 \\
Diaphragm & 1 & $11(2)$ \\
Pancreas & 0 & 1 \\
\hline
\end{tabular}

static liver involvement, and one had diffuse peritoneal seedlings with minimal ascites. Intravenous contrast was not used in any of these patients, but we now feel that it is of value in CT scanning, particularly if the ultrasound scan indicates any abnormality in liver echogenicity. ${ }^{12}$ We would now recommend using a single bolus intravenous contrast injection, to aid assessment of liver status and vascular invasion.

CT assessment of lymph node involvement is unreliable in this study. Results from previous studies indicate accuracy figures ranging from $50 \%{ }^{13}$ to $91 \%,{ }^{14}$ although the latter group confined their analysis to advanced gastric cancer. It is accepted that the only possible criterion for positive node involvement on CT is node size. The lower limit of resolution for both our scanners is $5 \mathrm{~mm}$. Therefore (using the information provided from a study of normal lymph nodes ${ }^{9}$ ) we decided that nodes in the chest greater than $5 \mathrm{~mm}$ in diameter should be termed positive and in the abdomen nodes greater than $9 \mathrm{~mm}$ should be classified as positive. However, it is well recognized that micrometastases may occur in normal sized glands and many enlarged glands may simply be due to reactive hyperplasia; ${ }^{9}$ this feature was confirmed in our study. Using our assessment criteria, the predictive values for a positive result regarding nodal status were 0.57 above the diaphragm and 
0.81 below the diaphragm. Specificity therefore was high $(92 \%, 85 \%)$ indicating that negative findings are usually correct; however, sensitivity was low $(36 \%, 43 \%)$. We feel our sensitivity results could be improved if we classified nodes in the abdomen smaller than $9 \mathrm{~mm}$ as positive as this would obviously include those normal sized nodes with microscopic invasion.

Previous studies ${ }^{12,13}$ have indicated that $\mathrm{CT}$ is not accurate either in terms of sensitivity or specificity regarding contiguous organ invasion. Several factors are involved, the paucity of mediastinal fat in these patients who are usually cachectic makes tumour definition unreliable; cardiac movement may also further interfere with interpretation. In our study the inaccuracies within the chest were related to aortic invasion. In 2 of our patients our findings would be correctly reclassified if we were to consider the possibility of invasion below a tumour/aortic circumference angle of 90 degrees. In summary, for invasion our results show a predictive value for positive results of 0.40 above the diaphragm, and 0.66 below the diaphragm; as with nodal involvement sensitivity is low but specificity is high.

In conclusion, we have found $\mathrm{CT}$ to be more accurate below the diaphragm, and to be more precise in assessing invasion than nodal status. However, although our numbers are small we have shown that specificity for both node involvement and invasion is high, indicating CT reliability for negative findings above and below the diaphragm. From our findings we feel that patients with a suggested positive result for node involvement should certainly not be denied surgery. A positive result for invasion is also not a contraindication to surgery except in those patients with evidence of tracheal or bronchial infiltration who from our results are likely to be true positives although admittedly our numbers in these groups are small. Patients in whom possible aortic or diaphragmatic invasion is suggested should be placed in the indeterminate category, and should not be denied surgery unless there is metastatic disease or a medical contraindication to operation.

CT is therefore of some value in assessing patients with oesophageal cancer, it confers some advantage over more standard radiological procedures. If used in combination with other investigations and the results are carefully interpretated it does aid in the overall management of these patients.

\section{References}

1. Ong, G.B., Laim, K.H., Wong, J. \& Lim, T.K. Factors influencing morbidity and mortality in oesophageal carcinoma. J Thorac Cardiovasc Surg 1978, 76: 745-754.

2. Postlethwait, R.W. Surgery of the Oesophagus. AppletonCentury-Crofts, New York, 1978, pp. 341-362.

3. Giluli, R. \& Lignoux, M. Treatment of carcinoma of the oesophagus: a retrospective study of 2400 patients. Ann Surg 1980, 192: 44-52.

4. Mortel, C.G. Carcinoma of the oesophagus: is there a role for surgery? The case against surgery. Ann J Dig Dis 1978, 23: 735.

5. Eftinck, M., Schattenkeck, H., Obertop, H. et al. Survival after carcinoma of the oesophagus. Br J Surg 1987, 74: $165-168$.

6. Orringer, M.B. Trans thoracic versus transhiatal esophagectomy. What difference does it make? Ann Thor Surg 1987, 44: $116-118$.

7. Daffner, R.H. Computed tomography of the oesophagus. CRC Crit Rev Diagn Imaging 1981, 14: 192-242.

8. Heitzman, E.R., Goldwin, R.L. \& Proto, A.V. Radiologic analysis of the mediastinum utilizing computed tomography. Radiol Clin North Am 1977, 15: 309-329.

9. Picus, D., Balfe, D.M., Koehler, R.E., Roper, C.L. \& Owel, J.W. Computed tomography in the staging of oesophageal carcinoma. Radiology 1983, 146: 433-438.

10. Husband, J.E. Role of CT scanner in the management of oesophageal cancer. Br Med J 1985, 146: 433-438.

11. Hennessy, T.P. Choice of treatment in carcinoma of the oesophagus. Br J Surg 1988, 75: 3-4.

12. Salonem, O., Kirisaari, L., Standevtskjold-Nordenstram, G., Somer, K. \& Virkkunen, P. Computed tomography in staging of oesophageal carcinoma. Scand J Gastroenterol 1987, 72: 65-68.

13. Thompson, W.M., Halvorsen, R.A., Foster, W.L., Wellford, M.E., Postlethwait, R.W. \& Korobkin,M. Computed tomography for staging oesophageal and gastro-oesophageal cancer. Am J Radiol 1983, 290: 527-530.

14. Dehn, T.C.B., Reznick, R.H., Nockler, I.B. \& White, F.E. The pre-operative assessment of advanced gastric cancer by computed tomography. Br J Surg 1984, 71: 413-417. 